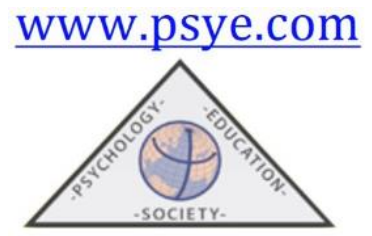

\title{
Inteligencia y experiencias tempranas adversas. ¿Relación con el apego y el autoconcepto adolescente?
}

\author{
Silveria Cambil Calderón y Milagros Fernández-Molina \\ Universidad de Málaga \\ Recibido el 15 de mayo de 2020. Aceptado el 17 de abril de 2021
}

\begin{abstract}
RESUMEN: El apego, el autoconcepto, la inteligencia y las experiencias tempranas adversas son factores fuertemente relacionados con el ajuste escolar y personal en la adolescencia, pero no hay estudios donde se analicen grupos que difieran en tales características. En este trabajo hemos estudiado la relación con el rendimiento académico en dos grupos de 30 adolescentes cada uno con $50 \%$ de chicos y chicas, con edades comprendidas entre los 13 y los 18 años $(M=13.9 ; D T=1.242)$, con necesidades específicas de apoyo educativo por privación sociocultural (adoptados y acogidos), y adolescentes por altas capacidades intelectuales. Los resultados muestran que ambos grupos difieren en la historia escolar, el rendimiento académico, la dimensión académica del autoconcepto y el porcentaje de sujetos en apego inseguro preocupado. Las dimensiones más relacionadas con el apego inseguro han sido el autoconcepto familiar y emocional, mientras que el apego seguro estuvo relacionado positivamente con la dimensión académica del autoconcepto. El rendimiento académico estuvo relacionado también con el autoconcepto académico y familiar, y con el traumatismo.
\end{abstract}

Palabras clave: adopción; adolescencia; cognición; personalidad.

\section{Intelligence and early adverse experiences: Relationship to adolescent attachment and self-concept}

ABSTRACT: Attachment, self-concept, intelligence and early adverse experiences are factors strongly related to school and personal adjustment in adolescence, but there are usually no studies where groups that differ in such characteristics are analyzed. In this work we have studied the relationship with academic performance in two groups of 30 adolescents each, 50\% girls and boys, 13 to 18 years old $(M=13.9 ; S D=1.242)$, with specific educational support needs due to socio-cultural deprivation (adopted and foster care) and high intellectual abilities. The results show that both groups differ in school history, academic performance, the academic dimension of selfconcept and the percentage of subjects in insecure attachment. The dimensions most related to insecure attachment have been family and emotional self-concept, while secure attachment was positively related to the academic dimension of self-concept. Academic performance was also related to academic and family self-concept, and trauma.

Keywords: Adoption; adolescence; cognition; personality. 


\section{Introducción}

El ajuste escolar se produce cuando el alumno tiene competencias para afrontar las tareas curriculares y de aceptación social (De la Orden y González, 2005; González-Barberá, 2004) siendo el alumnado con necesidades específicas de apoyo educativo (NEAE, en adelante) aquel que, por diferentes causas, puede presentar mayor riesgo. Los chicos y chicas procedentes del sistema de protección social, adoptados y acogidos (AA, en adelante), y los que tienen altas capacidades intelectuales (AACC, en adelante) son alumnado NEAE, aunque la naturaleza y el origen de sus necesidades y del riesgo de desajuste escolar sea distinto. Por ello, podríamos decir que los alumnos con mayor CI y el alumnado que ha sufrido situaciones carenciales en la infancia temprana son poblaciones de pronóstico escolar opuesto. Efectivamente, la inteligencia y las experiencias tempranas adversas son factores que predicen el ajuste escolar y el desarrollo académico (Díaz, 2018; González Barberá, 2004; Pérez Sánchez et al., 2007; Wai y Allen, 2019), pero otros aspectos emocionales, como el vínculo de apego (FernándezMolina, 2011), y personales, como la eficacia autorregulatoria, la motivación académica y el autoconcepto (Fernández Lasarte et al., 2019; Mammadov et al., 2018) deben ser tenidos en cuenta. En este trabajo pretendemos caracterizar los patrones de apego y autoconcepto de estos dos grupos de alumnado y comparar la relación entre apego, autoconcepto y rendimiento académico entre adolescentes altamente inteligentes, no considerados a priori población de alto riesgo, y adolescentes con historias tempranas adversas, analizando estas dos poblaciones en una etapa evolutivamente sensible al desajuste escolar, como es la adolescencia.

\section{Apego y su relación con el ajuste escolar}

Mientras que el apego ha sido muy analizado en los menores AA, no ocurre lo mismo con el alumnado NEAE por AACC (Dijkstra et al., 2017; Fernández-Molina, 2011; Wellisch, 2010). Los niños que, especialmente durante los 3 primeros años de vida, han sido institucionalizados o maltratados, que han vivido rupturas sucesivas con su figura de referencia afectiva o cuando esta presentaba falta de disponibilidad o inestabilidad, pueden desarrollar trastorno de apego o una mayor prevalencia de apego inseguro (Ainsworth y Bell, 1969; Bowlby, 1969/1982; Muela et al., 2012; Stronach et al., 2011; Van den Dries et al., 2009; Van Londen et al., 2007; Wilkins, 2012). La falta de apego seguro aumenta la probabilidad de que estos niños no superen adecuadamente los retos evolutivos y que, por tanto, no alcancen el desarrollo óptimo y que tengan bajo rendimiento académico (Cicchetti et al., 2006; Ramsdal et al., 2015). Así, distintas investigaciones apuntan a la presencia en estos niños de un continuo de dificultades que van desde carencias en las habilidades básicas para el aprendizaje, como el habla, la comprensión, la atención sostenida, la capacidad de esfuerzo personal y la dificultad en mecanismos de autorregulación (Hidalgo, 2010), hasta patologías neuropsicológicas, como retrasos del desarrollo, trastornos de la conducta y TDAH, autismo y problemas neurosensoriales (Enríquez et al., 2017; Maguire et al., 2015; Mansilla et al., 2018; Palacios et al., 2011; Rodriguez y Guzmán, 2019). La encuesta realizada por Petales España en el año 2019, apunta en la misma dirección y refleja que, de los menores AA, el 56\% tiene o tuvo reconocidas Necesidades Educativas Especiales. De ellos el 23\% tenía diagnosticado TDAH, el 21\% Trastorno Reactivo del Apego y el 15\% Trastorno Oposicionista Desafiante. De la misma forma, el establecimiento de un patrón de apego seguro influye positivamente en los procesos cognitivos infantiles en funciones frontales y ejecutivas, concretamente en las tareas que implican la memoria de trabajo, control inhibitorio y planificación de la conducta hacia metas, entre otras (Halpern y Muriel, 2012; O’Connor y McCartney, 2007).

Como hemos comentado, el estudio de la vinculación afectiva en población AACC apenas ha tenido interés, y la influencia mutua entre alta capacidad y estilo de apego y el impacto del apego 
inseguro sobre la alta capacidad ha sido insuficientemente examinada, pudiendo ser el estilo de apego un factor limitante en el desarrollo del potencial de esta población (Dijksta et al., 2017; Wellisch, 2010). Sin embargo, se asume que la inteligencia puede actuar como un factor personal de protección para el desarrollo de apego seguro incluso en ambientes tempranos difíciles (ADIMA, 1997; Barudy, 2014) ya que estos chicos y chicas presentan características facilitadoras para el desarrollo del apego seguro, como curiosidad y persistencia, alta preocupación por los demás, altos niveles de empatía, inteligencia social o desarrollo lingüístico avanzado (Wellisch, 2010). Por otra parte, algunos estudios hipotetizan que debe haber población AACC con patrones de apego inseguro al constatar que no hay equivalencia entre las tasas de apego seguro encontradas en población general y las tasas de AACC.

\section{El autoconcepto y su relación con el ajuste escolar}

El autoconcepto es definido actualmente como un esquema cognitivo, organizado y estructurado, que contiene la totalidad de las creencias de un individuo sobre sus propias características, así como también roles, valores y metas personales (Campbell et al., 1996; Stinson et al., 2008) y es un factor personal vinculado al bajo rendimiento, de forma que un bajo autoconcepto se asocia con menores resultados académicos, y asociado positivamente, en cambio, al locus de control interno, a expectativas adecuadas hacia los estudios y consecución de metas académicas (Álvarez et al., 2015; Rodríguez et al., 2017; Rodriguez y Guzman, 2019; Timmerman et al., 2017). También se ha encontrado efecto de género y de etapa evolutiva, ya que en algún estudio se observa un mayor deterioro evolutivo del autoconcepto académico en la adolescencia media de chicas, comparada con etapas anteriores y posteriores (Fernández Lasarte et al., 2019).

El estudio del autoconcepto en menores del sistema de protección social ha estado más centrado en la construcción de la identidad y la búsqueda de los orígenes o los niveles de autoestima que en el propio autoconcepto (Salas et al., 2015). La investigación considera que la autoestima de la población AA es un aspecto esencial relacionado con la aparición de problemas en la infancia y adolescencia ya que aquellos niños que experimentan protección y seguridad dentro del contexto familiar desarrollan un sentido de sí mismos más positivo (Kim y Cicchetti, 2006). La crítica y el rechazo parental, por tanto, incrementa los problemas en la autoestima infantil (Salas et al., 2015).

En el alumnado AACC se ha encontrado puntuaciones en autoconcepto general ligeramente superiores a las de los niños de inteligencia media (Hoge y Renzulli, 1993) y altos niveles de autoestima (Fernández-Reyes, 2005), aunque en cambio las dimensiones personal y social del autoconcepto no suelen ser tan elevadas (Galindo et al., 1999). Existe la falsa creencia de que los estudiantes AACC son inherentemente motivados y académicamente curiosos, en cambio, se ha visto cómo ciertas medidas educativas que se toman con el alumnado AACC, como la aceleración o flexibilización, afectan al autoconcepto teniendo el alumnado AACC flexibilizado mejor autoconcepto escolar y matemático (Mammadov et al., 2018). El tipo de perfil intelectual también se ha vinculado al autoconcepto ya que se ha encontrado que los niños con alta capacidad "analíticos" parecen tener mayores niveles de memoria de trabajo y de motivación a diferencia de los niños con alta capacidad "creativos" (Gubbels et al., 2008). Además, la tendencia a la baja tolerancia a la frustración o el perfeccionismo, tan prototípicos del alumnado AACC (Fernández-Reyes, 2005), podría derivarse de dificultades en el autoconcepto y la autoestima.

En conclusión, la importancia de analizar las relaciones entre inteligencia, autoconcepto, apego y rendimiento académico en alumnado adolescente con NEAE no es únicamente por examinar la relación entre estos aspectos, o por encontrar el peso de estos factores sobre el ajuste escolar personal de estudiantes AA y con AACC. Existe la necesidad de prestar atención a la adolescencia media, como periodo evolutivo de riesgo para el ajuste escolar, ya que se ha encontrado peor autoconcepto académico 
en estas edades incluso en adolescentes que en la infancia media o en la adolescencia temprana presentaban indicadores adecuados en la construcción de su autoconcepto (Fernández Lasarte et al., 2019), y también por la relación entre el autoconcepto y la actuación didáctica docente (De la Orden y González, 2005; Gil Flores, 2017).

\section{Metodología}

\section{Participantes}

Los participantes de este estudio son 60 adolescentes, 30 adoptados y acogidos con un nivel intelectual dentro de la media y con experiencias tempranas adversas (Grupo AA) y 30 adolescentes sin historia previa adversa y nivel intelectual alto (Grupo AACC), con edades comprendidas entre los 13 y los 18 años, y que cursan la Educación Secundaria Obligatoria. El grupo AA está compuesto por adolescentes adoptados o acogidos de distintas comunidades autónomas (Aragón, Andalucía, Principado de Asturias, Castilla la Mancha, Castilla León, Cataluña, Extremadura, Galicia, La Rioja, Madrid, Murcia y Navarra), cuyas familias son socias de la asociación Petales (Asociación de apoyo y ayuda mutua para familiares y personas que presentan trastornos de apego), todos ellos con una historia de desprotección previa a la adopción o acogimiento. El grupo AACC procede de un programa de mentorías universitarias para alumnado con altas capacidades intelectuales.

\section{Instrumentos}

Datos personales y sociodemográficos. Los datos referentes a las características de los participantes, así como los referentes a la historia de vida del menor, fueron obtenidos mediante la cumplimentación del Cuestionario de historia escolar de adolescentes (Cambil Calderón y FernándezMolina, 2019), concretamente de los apartados "Datos de identificación" e "Historia de vida".

Historia escolar. Los datos referentes a la edad de escolarización del menor, si asistió a educación infantil, cambios de colegio, cursos repetidos, identificación de NEAE, tipo de escolarización, etc. fueron obtenidos mediante el Cuestionario de historia escolar de adolescentes (Cambil Calderón y Fernández-Molina, 2019), concretamente el apartado "Historia escolar".

Autoconcepto. Los menores cumplimentaron la escala multidimensional de autoconcepto forma 5 (AF-5, García y Musitu, 1999). Esta escala es aplicable a partir de los 10 años y mide el autoconcepto en 5 dimensiones: académico/laboral, social, emocional, familiar y físico. La escala consta de 30 ítems, que son frases a las que se debe puntuar del 1 al 99, según el grado de acuerdo con lo que afirman.

Rendimiento académico. Se midió a través de las calificaciones de los adolescentes, ya que en los Cuestionarios de historia escolar de adolescentes (Cambil Calderón y Fernández-Molina, 2019) se solicitaba completar una tabla con las notas obtenidas por el alumno durante el primer y segundo cuatrimestre del curso académico 2018/2019.

Apego. Los menores cumplimentaron la escala CaMir-R (Balluerka et al., 2014). Esta escala consta de 7 dimensiones, con las que se puede evaluar tres estilos de apego: las dimensiones seguridad, disponibilidad y apoyo de las figuras de apego, evalúa el apego seguro; la dimensión interferencia de los padres, evalúa el apego inseguro preocupado; la dimensión autosuficiencia y rencor hacia los padres evalúa el estilo de apego inseguro evitativo, y, por último, la dimensión traumatismo infantil, puede ser un indicador de apego desorganizado (Lacasa y Muela, 2014). La escala consta de 32 ítems y puede ser aplicada entre los 13 y los 23 años. Si la puntuación en las distintas dimensiones es superior a 50 se considera alta. 


\section{Procedimiento}

Los datos de los adolescentes AA fueron recogidos mediante la cumplimentación de cuestionarios facilitados a los adolescentes y sus progenitores. Las familias fueron contactadas mediante un comunicado que hizo el presidente de la asociación Pétales, en la página web de la asociación, solicitando la colaboración de familias con hijos AA, que estuviesen en el instituto y tuviesen más de 13 años. Las familias que aceptaron colaborar en el estudio se pusieron en contacto con las investigadoras y facilitaron sus datos. Todos los cuestionarios estaban dispuestos en sobres prefranqueados, que fueron enviados por correo postal a las familias entre el 7 y el 9 de mayo del 2019. Esta recogida de datos se hizo siguiendo los estándares de procedimiento recogidos en el convenio entre empresas y asociaciones y la Universidad de Málaga para la realización de Trabajos Fin de Máster en la modalidad de colaboración con Empresas externas.

Los datos del grupo AACC fueron cumplimentados por los adolescentes y sus progenitores durante las sesiones de los talleres del programa de Mentorías, previa firma de un consentimiento informado para los progenitores y un documento de asentimiento informado para los adolescentes, según las indicaciones del Comité de Ética de la Universidad de Málaga que aprobó el procedimiento del estudio en el contexto de la solicitud de proyectos de investigación del Plan Nacional de Investigación.

\section{Análisis de Datos}

Se calcularon los estadísticos descriptivos para el grupo completo de participantes y para cada grupo por separado. Se realizaron correlaciones parciales a través de la prueba $r$ de Pearson para las puntuaciones en apego, autoconcepto, rendimiento académico y edad. Para establecer diferencias de medias entre ambos grupos de adolescentes se utilizó la prueba $t$ de student para muestras independientes tomando como nivel de significación aceptado $p<.05$. Para conocer el tamaño del efecto de las diferencias se calculó $d$ ' de Cohen (1992) (efecto pequeño: $\geq .20 d \leq .49$; efecto moderado: $\geq .50 d \leq$ .79; efecto grande: $\geq .80$ ). Igualmente se aplicó la prueba chi-cuadrado para la variable de presencia o ausencia de NEA. Se hizo uso del software de análisis de datos IBM SPSS statistics, en su versión 25.

\section{Resultados}

\section{Datos sociodemográficos e historia escolar}

En la Tabla 1 aparecen los estadísticos descriptivos referentes a la edad, sexo y trayectoria escolar de los adolescentes. Destacan los resultados que caracterizan la historia escolar del grupo AA: entrada tardía al sistema educativo (4.25 años), no asistencia al primer ciclo de la escuela infantil (75\%), repetición de curso ( 0.75 cursos repetidos) y presencia de necesidades educativas especiales (NEE) o dificultades de aprendizaje (DA) (28.6\%). Para estas últimas variables se realizó una prueba chicuadrado, que indicó diferencias significativas entre los grupos $\left(\chi^{2}(1,60)=5.27, p=.022\right)$, lo que muestra la relación entre la situación de adopción-acogimiento con la presencia de NEE y DA.

\section{Tabla 1}

Estadísticos descriptivos de la Historia escolar

\begin{tabular}{lcccc}
\hline & & Total & $A A$ & $A A C C$ \\
\hline Edad $M(D T)$ & & $13.9(1.24)$ & $14.29(0.3)$ & $13.56(0.31)$ \\
\hline Género & & & & \\
\hline & Chicas & $50 \%$ & $64.3 \%$ & $37.5 \%$ \\
\hline & Chicos & $50 \%$ & $35.7 \%$ & $62.5 \%$ \\
\hline
\end{tabular}




\begin{tabular}{|c|c|c|c|}
\hline Edad de entrada al sistema educativo $M(D T)$ & $3.63(1.06)$ & $4.25(1.25)$ & $3(0)$ \\
\hline Cursos repetidos $M(D T)$ & $0.38(0.51)$ & $0.75(0.5)$ & $0(0)$ \\
\hline \multicolumn{4}{|l|}{ Acudió a educación infantil } \\
\hline No & $12.5 \%$ & $25 \%$ & $0 \%$ \\
\hline Sí, primer ciclo & $50 \%$ & $25 \%$ & $75 \%$ \\
\hline Sí, segundo ciclo & $37.5 \%$ & $50 \%$ & $25 \%$ \\
\hline Acudió a atención temprana & $12.5 \%$ & $0 \%$ & $25 \%$ \\
\hline \multicolumn{4}{|l|}{ Tipo de instituto } \\
\hline Público & $75 \%$ & $50 \%$ & $100 \%$ \\
\hline Privado & $12.5 \%$ & $25 \%$ & $0 \%$ \\
\hline Concertado & $12.5 \%$ & $25 \%$ & $0 \%$ \\
\hline \multicolumn{4}{|l|}{ NEAE } \\
\hline NEE* & $13.3 \%$ & $28.6 \%$ & $0 \%$ \\
\hline DA* & $13.3 \%$ & $28.6 \%$ & $0 \%$ \\
\hline
\end{tabular}

Nota. $* \mathrm{X}^{2}=5.27, g l 1, p=.022 ; * * \mathrm{X}^{2}=30.0, g l 1, \mathrm{p}=.01$

\section{Rendimiento académico}

Las medias en las notas en el primer y segundo trimestre del curso 2018-2019 de ambos grupos están bastante distanciadas, siendo de $6.67(D T=1.99)$ en el grupo completo, $5.08(D T=1.04)$ para el grupo AA y de $8.27(D T=1.19)$ para el grupo AACC. Los resultados de la prueba t de Student muestran diferencias estadísticamente significativas entre ambos grupos $(t(6,60)=4.023, p=.007)$, de forma que el rendimiento académico de los adolescentes AA es significativamente inferior al de los adolescentes AACC sin historia previa de acogida o adopción.

\section{Autoconcepto}

A continuación, en la Tabla 2 se muestran los resultados obtenidos de la prueba AF5 (García y Musitu, 1999): puntuaciones directas en cada categoría para el total y para cada grupo y puntuaciones en centiles, que permiten valorar más adecuadamente la posición en cada dimensión. Así, según las puntuaciones directas, la puntuación más alta correspondería a la dimensión familiar $(M=7.98)$ seguida de la dimensión académica $(M=6.89)$, siendo la más baja la del autoconcepto emocional $(M=5.14)$. En cambio, si observamos las puntuaciones centiles es la dimensión académica la que resulta más alta en el total de participantes. Hemos encontrado diferencias significativas en la dimensión de autoconcepto académico $(t(28,60)=2.39, p=.032)$ entre los menores adoptados y acogidos $(M=5.79)$ y los adolescentes AACC $(M=7.86)$. Es decir, el grupo de menores adoptados y acogidos obtuvieron un autoconcepto académico significativamente más bajo que el grupo de adolescentes con AACC $(d=$ 0.81). En cambio, no hay diferencias en las dimensiones social, emocional y familiar del autoconcepto.

Tabla 2

Estadísticos descriptivos en autoconcepto. Puntuaciones directas y Puntuaciones centiles

\begin{tabular}{lcccccc}
\hline & Total PD & Total PT & AA PD & AA PT & AACC PD & AACC PT \\
& M (DT) & M (DT) & M (DT) & M (DT) & M (DT) & M (DT) \\
\hline Académico & $6.89(2.54)$ & $65.27(35.3)$ & $5.79(2.89)$ & $51.5(39.8)$ & $7.86(1.76)$ & $77.31(26.43)$ \\
\hline Social & $6.59(2.06)$ & $39.6(31.6)$ & $6.36(2.02)$ & $35.93(32.6)$ & $6.75(2.14)$ & $42.81(31.55)$ \\
\hline Emocional & $5.14(2.23)$ & $46.9(32.8)$ & $4.74(2.39)$ & $42.5(34.1)$ & $5.49(2.09)$ & $50.7(32.17)$ \\
\hline
\end{tabular}




\begin{tabular}{lllllll}
\hline Familiar & $7.98(2.05)$ & $53.1(32.4)$ & $8.07(1.94)$ & $53.43(30.6)$ & $7.89(2.2)$ & $52.88(34.9)$ \\
\hline Físico & $6.66(2.13)$ & $60.6(30.1)$ & $7.01(2.11)$ & $66.86(30.4)$ & $6.35(2.17)$ & $55.2(29.75)$ \\
\hline
\end{tabular}

Apego

En la Tabla 3 aparecen los resultados de la aplicación del CaMir-R (Balluerka et al., 2014) con indicación de los estadísticos descriptivos distribuidos en los tres estilos de representaciones del apego (seguro e inseguro, y éste de tipo preocupado o de tipo evitativo) que permite la prueba. El $41.4 \%$ del grupo tiene apego seguro, aunque el mayor porcentaje se encuentra en el grupo AACC. En cambio, en el grupo AA encontramos mayor porcentaje de sujetos con apego preocupado. Las diferencias entre grupos no fueron significativas.

\section{Tabla 3}

Estadísticos descriptivos en la prueba CaMir-R. Porcentajes por tipo de apego. Media y desviación típica de las puntuaciones obtenidas

\begin{tabular}{lcccccc}
\hline & Total & M (DT) & AA & M (DT) & AACC & M (DT) \\
\hline Seguro & $41.4 \%$ & $43.13(16.14)$ & $30.8 \%$ & $38.43(16.38)$ & $50.0 \%$ & $46.95(15.39)$ \\
Preocupado & $24.1 \%$ & $53.44(10.24)$ & $38.5 \%$ & $53.1(10.68)$ & $12.5 \%$ & $53.71(10.21)$ \\
Evitativo & $34.5 \%$ & $53.84(10.83)$ & $30.8 \%$ & $53.88(9.17)$ & $37.5 \%$ & $54.78(12.30)$ \\
Traumatismo & & $57.7(16.78)$ & & $60.15(14.38)$ & & $55.70(18.72)$ \\
\hline
\end{tabular}

Como se observa en la Tabla 3, la media de los sujetos AACC es superior en apego seguro ( $M=$ 46.95), mientras que en el resto de las dimensiones es inferior a la del grupo de adolescentes AA, es destacable la poca distancia que hay entre las puntuaciones de los dos grupos en apego ansioso o preocupado $(M=53.71$ y $M=53.10$, respectivamente), y evitativo $(M=54.78$ y $M=53.88$, respectivamente). Asimismo, en la Tabla 3 se incluyen las puntuaciones T en la dimensión independiente de traumatismo que permite calcular la prueba.

No se encontraron diferencias significativas entre grupos al usar la prueba t de student, aunque según la interpretación de la prueba CaMir-R una puntuación superior a 50 es alta, por lo que las puntuaciones en apego seguro de la muestra en general serían bajas, y por el contrario alta en los distintos tipos de apego inseguro.

\section{Relaciones apego, autoconcepto y rendimiento académico}

Se calcularon las correlaciones bivariadas $r$ de Pearson que se muestran en la Tabla 4. Los tres tipos de apego inseguro se han relacionado significativamente con algunas de las dimensiones del autoconcepto. Los tres tipos de apego menos positivos han resultado estar negativamente relacionados con las dimensiones familiar, académico, y físico del autoconcepto, destacando que el evitativo ha sido el tipo de apego que más vinculaciones tiene. Las dimensiones más relacionadas con el apego inseguro han sido el familiar y el emocional. Así, a mayor apego preocupado, menor autoconcepto familiar, o a mayor apego evitativo menor autoconcepto académico, emocional, familiar y físico. De la misma forma, a mayor traumatismo, menor autoconcepto académico, emocional y familiar. Para finalizar, hemos encontrado relación directa entre el apego seguro y el autoconcepto académico que además es más alto en el grupo AACC. El rendimiento académico estuvo relacionado con el autoconcepto académico y familiar y negativamente con la dimensión traumatismo. 


\section{Tabla 4}

Correlaciones entre edad, apego, autoconcepto y rendimiento académico

\begin{tabular}{cccccccccccc}
\hline \multicolumn{1}{c}{1} & 2 & 3 & 4 & 5 & 6 & 7 & 8 & 9 & 10 & 11 \\
\hline 1 & 1 & & & & & & & & & & \\
\hline 2 & $-.41^{*}$ & 1 & & & & & & & & & \\
\hline 3 & .24 & -.21 & 1 & & & & & & & & \\
\hline 4 & $.48^{*}$ & -.53 & $.52^{*}$ & 1 & & & & & & & \\
\hline 5 & $.44^{*}$ & -.55 & $.59^{*}$ & $.59^{*}$ & 1 & & & & & & \\
\hline 6 & $-.37^{*}$ & .38 & -.20 & $-.37^{*}$ & $-.47^{*}$ & 1 & & & & & \\
\hline 7 & -.22 & .31 & -.08 & -.31 & -.32 & .30 & 1 & & & \\
\hline 8 & $-.44^{*}$ & .04 & -.30 & $-.39^{*}$ & $-.42^{*}$ & .34 & .20 & 1 & & \\
\hline 9 & $-.37^{*}$ & .34 & $-.53^{*}$ & $-.68^{*}$ & $-.50^{*}$ & $.58^{*}$ & .23 & $.46^{*}$ & 1 & & \\
\hline 10 & -.10 & .17 & -.26 & -.41 & -.34 & .50 & $.49^{*}$ & .33 & $.64^{*}$ & 1 & \\
\hline 11 & -.30 & .31 & -.11 & -.11 & $-.48^{*}$ & $.84^{*}$ & .06 & .34 & $.42^{*}$ & .24 & 1 \\
\hline
\end{tabular}

Nota: 1 = Edad; 2 = Apego seguro; 3 = Apego preocupado $4=$ Apego evitativo; $5=$ Traumatismo $; 6=$ Académico; $7=$ Autoconcepto Social; 8 = Autoconcepto Emocional; 9 = Autoconcepto Familiar; $10=$ Autoconcepto Físico; $11=$ Rendimiento Académico.

\section{Discusión}

El objetivo de este trabajo era caracterizar los patrones de apego y autoconcepto de dos grupos de alumnado NEAE que se diferenciaban en niveles intelectuales y en vivencias tempranas, y su relación con el rendimiento académico. Así, hemos comparado la relación entre apego, autoconcepto y rendimiento académico en adolescentes altamente inteligentes, no considerados a priori población de alto peor pronóstico de dificultades académicas, y adolescentes con historias de privación sociocultural, en los que la literatura ha identificado bajo rendimiento escolar, analizando estas dos poblaciones en una etapa evolutivamente sensible al desajuste escolar, como es la adolescencia.

En cuanto al historial escolar y al rendimiento académico, es destacable que, como cabía esperar, el grupo de adolescentes AA presenta una trayectoria escolar de mayor riesgo para el bajo rendimiento y un rendimiento académico significativamente más bajo que los del grupo AACC, estando estos resultados en consonancia con lo encontrado en la literatura referenciada (Díaz, 2018; González Barberá, 2004; Pérez Sánchez et al., 2007; Wai y Allen, 2019).

El patrón de apego seguro es el que observamos en la mayoría de los participantes, aunque las puntuaciones no son especialmente altas, ya que están por debajo de 50, y no podemos afirmar que, aunque sea mayor en el grupo AACC, esta diferencia sea significativa. Coherentemente, hemos encontrado una relación significativa positiva entre apego inseguro y evitativo y traumatismo y, que puntuaciones altas en apego seguro correlacionan inversamente con las de evitativo y traumatismo. Es sorprendente encontrar que, aunque el grupo de adolescentes AA tuviese puntuaciones más altas en las medidas de apego inseguro, estas estuvieron muy cercanas a las del grupo AACC en las dimensiones de apego ansioso y evitativo, siendo esperable que estas medidas estuviesen más distanciadas. Una posible explicación podría estar en los porcentajes esperados de apego ansioso y evitativo de la población general, aunque otra posible explicación estaría en las historias de vida de los adolescentes, en las que sería necesario profundizar. A pesar de las similitudes entre ambos grupos en apego ansioso y evitativo, en este estudio se puede constatar la diferencia entre los grupos en la dimensión traumatismo, aunque ésta no es significativa. Esta dimensión, sin ser medida del apego desorganizado, sí es indicador de este (Lacasa y Muela, 2014) y, por tanto, con la prudencia que exige, este resultado podría estar en consonancia con distintas investigaciones que afirman la prevalencia de apego desorganizado entre las poblaciones AA (Muela et al., 2012; Stronach et al., 2011; Van den Dries et al., 2009; Van Londen et al., 2007; Wilkins, 2012). 
En cuanto a las puntuaciones en autoconcepto, el grupo AA tiene un autoconcepto más bajo, sobre todo el académico, que ha sido el más asociado al bajo rendimiento (Álvarez et al., 2015; González-Barberá, 2004; Miñano y Castejón, 2011). La dimensión familiar del autoconcepto ha sido muy importante en nuestro estudio porque aquellos sujetos que tenían puntuaciones más altas también las tenían en académico, físico y emocional. En cambio, el autoconcepto social solo se vinculó con el físico, lo que es coherente dada la etapa adolescente que estudiamos. Finalmente, los tres tipos de apego inseguro se han relacionado significativamente con algunas de las dimensiones del autoconcepto y hemos encontrado relación directa entre el apego seguro y el autoconcepto académico que además es más alto en el grupo AACC. Estos resultados nos señalan la importancia del contexto familiar y de la construcción de relaciones afectuosas, disponibles y sensibles tanto en población AA como en AACC. Asimismo, pensamos que la eficacia en la gestión de la clase y de la enseñanza de un profesor podría actuar como protector sobre el autoconcepto académico de los alumnos. Es importante identificar estos patrones en la escuela para que el profesorado sea más sensible y eficaz y su actuación funcione como factor de protección. Sabemos que cuando un docente encarga trabajos diferentes a aquel alumnado con dificultades y/o a los que avanzan más deprisa, o hace un uso formativo de la evaluación y usa enfoques de aprendizaje individualizado, puede estar favoreciendo el desarrollo de estos aspectos, a la vez que afianza la relación de apego segura con su alumnado (O’Connor y McCartney, 2007).

\section{Limitaciones y futuras líneas}

Aunque los resultados apuntan en la dirección de la revisión teórica expuesta en la introducción, habría que continuar este estudio con un mayor número de participantes que nos permitan realizar análisis más potentes. Asimismo, hubiese sido interesante conseguir una medida directa del apego desorganizado y tener en cuenta la diversidad cognitiva en la población AACC, analizando la influencia del tipo de perfil intelectual, y la diversidad contextual y motivacional, analizando aspectos de la vida familiar y de los intereses y motivaciones de estos adolescentes.

\section{Conclusiones}

En definitiva, este estudio describe los patrones de apego y autoconcepto de dos grupos de alumnado NEAE que se diferencian en niveles intelectuales y en vivencias tempranas Los adolescentes acogidos y adoptados han presentado más factores de riesgo, un autoconcepto académico más bajo y peor rendimiento académico, aunque el patrón de apego predominante en los dos grupos estudiados ha sido el de apego seguro. Se ha encontrado relación directa entre apego seguro y autoconcepto académico, y entre éste y el rendimiento escolar, lo que ratifica la importancia de las dinámicas familiares sobre el éxito en los aprendizajes. Se defiende la necesidad de que el profesorado sea sensible a las necesidades de estas dos poblaciones, alumnado con altas capacidades y alumnado con historias tempranas adversas, en el sentido de ayudarles a mejorar el autoconcepto académico durante su estancia en el sistema educativo.

\section{Referencias}

ADIMA (1997). Guía de atención al maltrato infantil. Asociación para la defensa de la infancia y la prevención del maltrato.

Ainsworth, M. D. S., y Bell, S. M. (1969). Some contemporary patterns in the feeding situation. En A. Ambrose (Ed,), Stimulation in early infancy (pp. 133-170). Academic Press. 
Álvarez, A., Suárez, N., Tuero, E., Núñez, J. C., Valle, A., y Regueiro, B. (2015). Implicación familiar, autoconcepto del adolescente y rendimiento académico. European Journal of Investigation in Health, Psychology and Education, 5(3), 293-311.

Balluerka, N., Lacasa, F., Gorostiaga, A., Muela, A., y Pierrehumbert, B. (2011). Versión reducida del cuestionario CaMir (CaMir-R) para la evaluación del apego. Psicothema, 23(3), 486-494.

Barudy, J. (2014). Infancia en contextos de riesgo, traumas infantiles y resiliencia. En A.S. Jiménez Hernández, A. Pantoja, J.J. Leiva Olivencia y E. Moreno (coords.). Infancia en contextos de riesgo. Editorial GEU.

Bowlby, J. (1969/1982). Attachment and loss: Vol. 1. Attachment. Basic Books.

Cambil Calderón, S. M., y Fernández-Molina, M. (2019). Cuestionario de historia escolar de adolescentes versión padres. Universidad de Málaga. Recuperado de https://www.safecreative.org/work/1904260763110-cuestionario-de-historia-escolaradoptados-version-padres-cambil-calderon-fernandez-molina-2019-

Campbell, J. D., Trapnell, P. D., Heine, S. J., Katz, I. M., Lavallee, L. F., y Lehman, D. R. (1996). Selfconcept clarity: measurement, personality correlates, and cultural boundaries. Journal of Personality and Social Psychology, 70(1), 141-156. http://dx.doi.org/10.1037/0022$\underline{3514.70 .1 .141}$

Cicchetti, D., Rogosch, F. A., y Toth, S. L. (2006). Fostering secure attachment in infant in maltreating families through preventive interventions. Development and Psychopathology, 18, 623-649.

De la Orden, A., y González, C. (2005). Variables que discriminan entre alumnos de bajo y medio-alto rendimiento académico. Revista de Investigación educativa, 23(2), 573-599.

Díaz, A. L. (2018). Factores personales, familiares y académicos que afectan al fracaso escolar en la Educación Secundaria. Electronic Journal of Research in Education Psychology, 1(1), 43-66.

Dijkstra, P., Barelds, D. P. H., Ronner, S., y Nauta, A. P. (2017). Intimate relationships of the intellectually gifted: Attachment style, conflict style and relationship satisfaction among members of the Mensa society. Marriage \& Family Review, 53(3), 262-280. https://doi.org/(...)1494929.2016.1177630

Enríquez, E. B., Deaño, M. D., Blanco, J. C. B., y Andión, I. R. (2017). Problemas de conducta y académicos según el itinerario vital en adopción. Revista de estudios e investigación en psicología y educación 1. https://doi.org/10.17979/reipe.2017.0.01.2604

Fernández Lasarte, O., Goñi, E., Camino. I., y Zubeldia, M. (2019). Ajuste escolar y autoconcepto académico en la Educación Secundaria. Revista de Investigación educativa, 37(1), 2163-179. https://doi.org/10.6018/rie.37.1.308651

Fernández-Molina, M. (2011). Adopción, desarrollo y aprendizaje. Padres y Maestros/Journal of Parents and Teachers, 339, 13-17.

Fernández-Reyes, M.T. (2005). Desarrollo socioemocional. Curso avanzado de Altas Capacidades Intelectuales. Documento original sin publicar.

Galindo, A., Martínez, F., y Arnáiz, P. (1999). El autoconcepto en el superdotado. En M.D. Prieto (coord.), Identificación, evaluación y atención a la diversidad del superdotado (pp. 121-134). Ediciones Aljibe.

García, F., y Musitu, G. (1999). AF5: Autoconcepto Forma 5. TEA Ediciones.

González Barbera, C. (2004). Factores determinantes del bajo rendimiento académico en educación secundaria (tesis doctoral). Universidad Complutense de Madrid, Servicio de Publicaciones.

Gubbels, J., Segers, E., y Verhoeven, L. (2018). How children's intellectual profiles relate to their cognitive, socio-emotional, and academic functioning. High Ability Studies, 29(2), 149-168. https://doi.org/10.1080/13598139.2018.1507902 
Halpern, G., y Muriel, E. (2012). Las malas funciones ejecutivas y la salud mental en el desarrollo. Revista Chilena de Neuropsiquiatría, 50(3), 147-148.

Hidalgo, S. (2010). Evaluación psicopedagógica, necesidades y pautas de mejora educativa en la adopción internacional. En Fernández-Molina, M. (coord.), Acogimiento y adopción en la escuela (pp. 207-218). Fundación General de la Universidad de Málaga.

Hoge, R. D., y Renzulli, J. S. (1993). Exploring the link between giftedness and self-concept. Review of Educational Research, 63(4), 449-465. https://doi.org/10.3102/00346543063004449

Hoogeveen, L., Janet G., van Hell, L., y Verhoeven, L. (2009). Self-Concept and Social Status of Accelerated and Nonaccelerated Students in the First 2 Years of Secondary School in the Netherlands. Gifted Child Quarterly, 53(1), 50-67. https://doi.org/10.1177/0016986208326556

Maguire, S. A., Williams, B., Naughton, A. M., Cowley, L. E., Tempest, V., Mann, M. K., y Kemp, A. M. (2015). A systematic review of the emotional, behavioural and cognitive features exhibited by school-aged children experiencing neglect or emotional abuse. Child: care, health and development, 41(5), 641-653.

Mammadov, S., Cross, T., y Ward, T. (2018) The Big Five personality predictors of academic achievement in gifted students: Mediation by self-regulatory efficacy and academic motivation, High Ability Studies, 29(2), 111-133. http://doi.org/10.1080/13598139.2018.1489222

Mansilla Yuguero, M., Olaya Meza, C., y Picazo Ortega, A. (2018). ¿Qué papel tiene el apego en la aparición de dificultades y fortalezas en menores en acogimiento residencial? Psychology, Society \& Education, 10(2), 163-171.

Muela, A., Torres, B., y Balluerka, N. (2012) Estilo de apego y psicopatología en adolescentes víctimas de maltrato infantil. Infancia y Aprendizaje, 35(4), 451-469. http://doi.org/10.1174/021037012803495294

O'Connor, E., y McCartney, K. (2007). Attachment and cognitive skills: An investigation of mediating mechanisms. Journal of Applied Developmental Psychology, 28, 458-476. http://doi.org/10.1016/j.appdev.2007.06.007

Palacios, J., Román, M., y Camacho, C. (2011). Growth and development in internationally adopted children: extent and timing of recovery after early adversity. Child: Care, Health and Development, 37(2), 282-288.

Pérez Sánchez, Beltrán, J., González Barberá, C. y Adrados Cuesta, J. (2007) Gender Differences in Intelligence and Achievement in Gifted Spanish Children. Gifted and Talented International, 22(2), 96-104. http://doi.org/10.1080/15332276.2007.11673500

Petales. (2019). ¿Cuál es la realidad de las familias de Petales en España? Una mirada a nuestras cifras. Recuperado de http://petales.es/cual-es-la-realidad-de-las-familias-de-petales-en-cifras/

Ramsdal, G., Bergvik, S., y Wynn, R. (2015). Parent-child attachment, academic performance and the process of high-school dropout: A narrative review. Attachment \& Human Development, 17(5), 522-545. http://doi.org/10.1080/14616734.2015.1072224

Rodríguez Rodríguez, D., y Guzmán Rosquete, R. (2019). Rendimiento académico de adolescentes declarados en situación de riesgo. Revista de Investigación Educativa, 37(1), 147-162. http://doi.org/10.6018/rie.37.1.303391

Rodríguez, S., Piñeiro, I., Gómez-Taibo, M. L., Regueiro, B., Estévez, I., y Valle, A. (2017). An explanatory model of maths achievement: Perceived parental involvement and academic motivation. Psicothema, 29(2), 184-190. http://doi.org/10.7334/psicothema2017.32

Salas, M., García-Martín, M. A., Fuentes. M. J., y Bernedo, I. M. (2015). Children's Emotional and Behavioral Problems in the Foster Family Context. J. Child Fam Stud 24, 1373-1383. http://doi.org/10.1007/s10826-014-9944-1 
Stinson, D. A., Wood, J. V., y Doxey, J. (2008). Search of Clarity: Self-Esteem and Domains of Confidence and Confusion. Personality and Social Psychology Bulletin, September. http://doi.org/10.1177/0146167208323102

Stronach, E. P., Toth, S. L., Rogosch, F., Oshri, A., Manly, J. T., y Cicchetti, D. (2011). Child maltreatment, attachment security, and internal representations of mother and mother-child relationships. Child Maltreatment, 16(2), 137-145.

Timmerman, H., Toll, S., y Van Luit, J. (2017). The relation between math self-concept, test and math anxiety, achievement motivation and math achievement in 12 to 14-year-old typically developing adolescents. Psychology, Society \& Education, 9(1), 89-103.

Van den Dries, L., Juffer, F., Van Ijzendoorn, M. H., y Bakermans-Kranenburg, M. J. (2009). Fostering security? A meta-analysis of attachment in adopted children. Children and Youth Services Review, 31, 410-421.

Van Londen, W. M., Juffer, F., y Van Ijzendoorn, M. H. (2007). Attachment, cognitive, and motor development in adopted children: Short-term outcomes after international adoption. Journal of Pediatric Psychology, 32(10), 1249-1258.

Wai. J., y Allen, J. (2019). What Boosts Talent Development? Examining Predictors of Academic Growth in Secondary School Among Academically Advanced Youth Across 21 Years. Gifted Child Quarterly, 63(4) 253-272. http://doi.org/10.1177/001698621986904

Wellisch, M. (2010) Communicating Love or Fear: The Role of Attachment Styles in Pathways to Giftedness, Roeper Review, 32(2), 116-126, http://doi:10.1080/02783191003587900

Wilkins, D. (2012). Disorganised attachment indicates child maltreatment: how is this link useful for child protection social workers? Journal of Social Work Practice, 26(1), 15-30. 